\title{
A SOBOLEV ESTIMATE FOR THE ADJOINT RESTRICTION OPERATOR
}

\author{
YONGGEUN CHO, ZIHUA GUO, AND SANGHYUK LEE
}

\begin{abstract}
In this note we consider the adjoint restriction estimate for hypersurface under additional regularity assumption. We obtain the optimal $H^{s}-L^{q}$ estimates and their mixed norm generalizations. As applications we prove some weighted Strichartz estimates for the propagator $\varphi \rightarrow e^{i t(-\Delta)^{\alpha / 2}} \varphi, \alpha>0$.
\end{abstract}

\section{INTRODUCTION}

The Fourier extension operator (the adjoint of restriction operator) $R^{*}$ for the sphere is defined by

$$
R^{*} f(x)=\int_{\mathbb{S}^{d}} e^{i x \cdot \xi} f(\xi) d \sigma(\xi), \quad x \in \mathbb{R}^{d+1} .
$$

Here $\mathbb{S}^{d}$ denotes the unit sphere in $\mathbb{R}^{d+1}$ and $d \sigma$ is the induced Lebesgue measure on $\mathbb{S}^{d}$. The problem which is known as the restriction problem for the sphere is to determine the range of $p, q$ for which

$$
\left\|R^{*} f\right\|_{L^{q}\left(\mathbb{R}^{d+1}\right)} \leq C\|f\|_{L^{p}\left(\mathbb{S}^{d}\right)}
$$

holds. As can be easily seen by Knapp's example and the asymptotic expansion of $\widehat{d \sigma},(1.1)$ holds only if $q>\frac{2(d+1)}{d}$ and

$$
(d+2) / q \leq d(1-1 / p) .
$$

When $d=1$, (1.1) on the optimal range was obtained by Zygmund [29] (see [7] for an earlier result due to Fefferman and Stein [7]). It has been conjectured that the necessary condition is sufficient for (1.1) in higher dimensions but it still remains open. When $p=2$, the sharp boundedness is due to Tomas [24] and Stein [17]. The result beyond Stein-Tomas range was first obtained by Bourgain when $d=2$, and further progresses were made by the works of Wolff [27], Tao, Vargas and Vega 23, Tao and Vargas [22], and Tao [21]. (Also see [28, 13, 26, 14] for results regarding different types of hypersurfaces.) Recently, Bourgain and Guth [4] improved the range. Especially, when $p=q$, the estimate (1.1) was shown to be true for $p \in$ $\left(56 / 17, \infty\right.$ ] in $\mathbb{R}^{2+1}$ (see [4, p.1265]) and for $p \in\left(p_{\circ}(d), \infty\right]$ in $\mathbb{R}^{d+1}, d \geq 3$ where $p_{\circ}(d)=2+12 /(4 d+1-k)$ if $d+1 \equiv k(\bmod 3), k=-1,0,1$. Their result relies on the multilinear restriction estimate in Bennett, Carbery and Tao [2]. A further improvement in $\mathbb{R}^{2+1}$ which gives (1.1) for $p=q \in(3.25, \infty]$ was obtained by Guth [10.

2010 Mathematics Subject Classification. 42B10, 42B25.

Key words and phrases. Restriction estimate, Strichartz estimate. 
In this note, we consider the estimate (1.1) from a different perspective. Let us denote by $\mathcal{H}^{s}$ the $L^{2}$ Sobolev space of order $s$ on the sphere. The main purpose of this paper is to study the restriction estimate

$$
\left\|R^{*} f\right\|_{q} \leq C\|f\|_{\mathcal{H}^{s}}
$$

and to find the optimal range of $s, q$ for which (1.2) holds. When $s=0$, by the necessary condition and Tomas-Stein theorem (1.2) holds if and only if $q \geq 2(d+$ $2) / d$. It is natural to expect that the range of $q$ gets wider if $f$ is assumed to have an additional regularity, that is to say $s>0$. However, for $q \leq 2(d+1) / d$ this estimate fails because $\widehat{d \sigma} \notin L^{q}$. Hence, the estimate (1.2) is of interest for $q$ satisfying $2(d+1) / d<q<2(d+2) / d$. By the Knapp type example again, it can be shown (see the paragraph below Theorem 1.2) that (1.2) is possible only if

$$
s \geq s_{q}=s_{q}(d)=: \frac{d+2}{q}-\frac{d}{2} .
$$

The estimate (1.2) for $s=s_{q}$ can be deduced from the sharp restriction estimate (11.1) with $(d+2) / q=d(1-1 / p))$ by making use of the embedding $\mathcal{H}^{\alpha}\left(\mathbb{S}^{d}\right) \subset L^{p}\left(\mathbb{S}^{d}\right)$ with $\alpha \geq d / 2-d / p$ and $p \neq \infty$. Since we have the sharp restriction estimate for $d=1$, we get (1.2) for $4<q<6$ with the optimal regularity. In higher dimensions it seems natural to expect that the estimate (1.2) holds whenever $2(d+1) / d<q<$ $2(d+2) / d$ and (1.3) is satisfied. As it turns out, this is indeed the case and the problem is much easier than the restriction estimate (1.1). This is mainly due to the fact that the inequality (1.2) is based on $L^{2}$-spaces.

The following is our first result.

Theorem 1.1. Let $d \geq 1$ and $q<2(d+2) / d$. Then (1.2) holds if and only if $s \geq s_{q}$ and $q>2(d+1) / d$.

Mixed norm generalization. We now consider a more general class of operators. Let $\phi$ be a smooth function. Then we define an extension operator $E$ by

$$
E f(x, t)=E_{\phi} f(x, t)=\int e^{i(x \cdot \xi+t \phi(\xi))} a(\xi) f(\xi) d \xi, \quad(x, t) \in \mathbb{R}^{d} \times \mathbb{R},
$$

where $a$ is a smooth function supported in $B(0,1)$. We denote by $B(z, r)$ the ball of radius $r$ which is centered at $z$. Generalizing (1.2) we consider the estimate

$$
\|E f\|_{L_{t}^{q}\left(\mathbb{R}, L_{x}^{r}\left(\mathbb{R}^{d}\right)\right)} \leq C\|f\|_{H^{s}} .
$$

Here $H^{s}$ is the usual inhomogeneous Sobolev space of order $s$. If the hessian matrix $H \phi$ of $\phi$ is nonsingular on the support of $a$, then by the Strichartz estimate (1.4) holds for $s=0$ and $q, r$ satisfying $2 \leq q, r \leq \infty, d / r+2 / q \leq d / 2,(q, r, d) \neq(2, \infty, 2)$ (see [12]). Let us set

$$
s_{c}=s_{c}(r, q, d)=\frac{d}{r}+\frac{2}{q}-\frac{d}{2} .
$$

As a mixed norm generalization of (1.2) we obtain the following.

Theorem 1.2. Suppose $\operatorname{det} H \phi \neq 0$ on the support of a. If $2 \leq q, r<\infty, d / r+$ $2 / q>d / 2$ and $d / r+1 / q<d / 2$, then (1.4) holds whenever $s \geq s_{c}$. If $d / r+1 / q=d / 2$ and $q \neq 2$, then the weak type estimate $\|E f\|_{L_{t}^{q, \infty}\left(\mathbb{R}, L_{x}^{r}\left(\mathbb{R}^{d}\right)\right)} \leq C\|f\|_{H^{s_{c}}}$ holds. 
Theorem 1.1 is an obvious consequence of Theorem 1.2 as this can be easily shown by making use of proper coordinate patches (for example, see [20, Sec. 3, Ch. 4]). The estimate (1.4) is no longer true if $d / r+1 / q \geq d / 2$ because $E_{\phi}(1) \notin L_{t}^{q} L_{x}^{r}$, which follows from Lemma 2.1. And the regularity condition is also optimal since (1.4) fails if $s<s_{c}$. Indeed, let us take $f(\xi)=\lambda^{\frac{d}{2}} \eta(\lambda \xi)$ with a compactly supported smooth $\eta$ so that $\|f\|_{H^{s}} \lesssim \lambda^{s}$. Then it is easy to see that $|E f(x, t)| \gtrsim \lambda^{-\frac{d}{2}}$ if $|t| \leq c \lambda^{2}$ and $|x+\nabla \phi(0) t| \leq c \lambda$ for a small $c>0$. Hence (1.4) implies

$$
\lambda^{-\frac{d}{2}} \lambda^{\frac{d}{r}+\frac{2}{q}} \lesssim \lambda^{s}
$$

Letting $\lambda \rightarrow \infty$, we get the condition (1.3).

Remark 1. Since $E$ is a localized operator, in Theorem 1.2 we may replace $H^{s}$ by the homogeneous Sobolev space $\dot{H}^{s}$ provided that $0<s<d / 2$. The same is also true for Theorem 1.1. Indeed, it is easy to see that for $0<s<d / 2$

$$
\|f \beta\|_{H^{s}} \lesssim\|f\|_{\dot{H}^{s}}
$$

where $\beta \in C_{0}^{\infty}\left(\mathbb{R}^{d}\right)$.

Application to Strichartz estimates. The adjoint restriction estimates are closely related to the Strichartz estimates. Especially, various space time estimates for the Schrödinger operator can be deduced from restriction estimates for the paraboloid. (See [15] for a detailed discussion.) As applications of Theorem 1.2 we obtain some weighted Strichartz estimates on the range where the usual Strichartz estimates are not allowed.

Let $\alpha>0$ and $\alpha \neq 1$. We first consider the weighted Strichartz estimates for the fractional Schrödinger operator

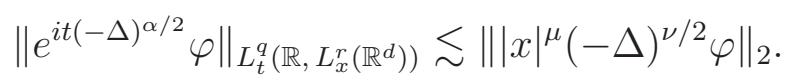

Corollary 1.3. Let $d \geq 1, \alpha>0, \alpha \neq 1$ and $2 \leq q, r<\infty$. If $d / r+2 / q>d / 2$ and $d / r+1 / q<d / 2$, then (1.6) holds provided that $\mu=s_{c}$ and $\nu=\frac{2-\alpha}{q}$.

We now consider the case $\alpha=1$, that is to say the wave operator. Let us set $s_{c}^{w}:=s_{c}(q, r, d-1)$.

Corollary 1.4. Let $d \geq 2, \alpha=1$ and $2 \leq q, r<\infty$. If $(d-1) / r+2 / q>(d-1) / 2$ and $(d-1) / r+1 / q<(d-1) / 2$, then (1.6) holds provided that $\mu=s_{c}^{w}$ and $\nu=\frac{1}{2}+\frac{1}{q}-\frac{1}{r}$.

Let $\Delta_{\omega}$ be the Laplace-Beltrami operator on the unit sphere $\mathbb{S}^{d-1} \subset \mathbb{R}^{d}$ which is given by $\Delta_{\omega}=\sum_{1 \leq i<j \leq d} \Omega_{i, j}^{2}, \Omega_{i, j}=\omega_{i} \partial_{j}-\omega_{j} \partial_{i}$. Then define a Sobolev norm $\|\cdot\|_{H_{s p h}^{\nu}}$ by setting

$$
\|f\|_{H_{s p h}^{\nu}}^{2}=\int_{0}^{\infty} \int_{S^{d-1}}\left|\left(1-\Delta_{\omega}\right)^{\nu / 2} f(r \omega)\right|^{2} d \sigma_{\omega} r^{d-1} d r .
$$

Let us consider the estimate

$$
\left\|(-\Delta)^{\gamma_{1} / 2} e^{i t \sqrt{-\Delta}} \varphi\right\|_{L_{t}^{q}\left(\mathbb{R}, L_{x}^{r}\left(\mathbb{R}^{d}\right)\right)} \leq C\|\varphi\|_{H_{s p h}^{\nu}}
$$

assuming the natural scaling invariant condition

$$
\gamma_{1}=\frac{1}{q}+\frac{d}{r}-\frac{d}{2}
$$


This type of inequality was studied by Sterbenz [19] to extend the range of admissible $q, r$ by making use of angular regularity (see [6, 5, 9] for related results and references therein). It is known ([19]) that the estimate (1.7) holds only if

$$
\nu \geq s_{c}^{w}, \quad \frac{d-1}{r}+\frac{1}{q}<\frac{d-1}{2} .
$$

Sterbenz [19] showed (1.7) for $\nu>s_{c}^{w}$. Our result enables us to obtain the estimate of the endpoint regularity when $q \geq r$.

Corollary 1.5. Let $d \geq 2$ and $2 \leq q, r<\infty$. If $q \geq r,(d-1) / r+2 / q>(d-1) / 2$ and $(d-1) / r+1 / q<(d-1) / 2$, then (1.7) holds provided that $\nu \geq s_{c}^{w}$ and (1.8).

In addition to ${ }^{\wedge}$ and ${ }^{\vee}$, we use $\mathcal{F}, \mathcal{F}^{-1}$ to denote Fourier, inverse Fourier transforms, respectively. The paper is organized as follows. In section 2 we provide a few preliminaries for the proof of Theorem 1.2. In section 3 we prove Theorem 1.2 and the proofs of Corollary 1.3, 1.4 and 1.5 are given in section 4 .

\section{Preliminaries}

For the proof of the estimate (1.4) we may assume that $\phi$ is close to a quadratic form. More precisely, let $\phi$ be a smooth function satisfying that det $H \phi$ is nonsingular. Then we may assume that

$$
\phi(\xi)=\frac{1}{2} \xi^{t} D \xi+\mathcal{E}(\xi),\|\mathcal{E}\|_{C^{L}(B(0,2))} \leq C \epsilon_{0}
$$

with a sufficiently small $\epsilon_{0}>0$ and a sufficiently large positive integer $L$ where $D$ is the diagonal matrix with nonzero entries \pm 1 .

Parabolic rescaling. Indeed, let $\xi_{0}$ be a point in $B(0,1)$. By decomposing a into finite number of smooth functions which are supported in small balls we need only to consider the localized operator

$$
\int e^{i(x \cdot \xi+t \phi(\xi))} a_{\xi_{0}, \epsilon_{0}}(\xi) f(\xi) d \xi
$$

where $a_{\xi_{0}, \epsilon_{0}}$ is a smooth function supported in $B\left(\xi_{0}, \epsilon_{0}\right)$. By Taylor expansion we have

$$
\phi(\xi)=\phi\left(\xi_{0}\right)+\nabla \phi\left(\xi_{0}\right) \cdot\left(\xi-\xi_{0}\right)+\frac{1}{2}\left(\xi-\xi_{0}\right)^{t} H \phi\left(\xi_{0}\right)\left(\xi-\xi_{0}\right)+O\left(\left|\xi-\xi_{0}\right|^{3}\right) .
$$

By discarding harmless factors, translation $\xi \rightarrow \xi+\xi_{0}$ and the linear change of variables $(x, t) \rightarrow\left(x+t \nabla \phi\left(\xi_{0}\right), t\right)$ we may assume

$$
\phi(\xi)=\frac{1}{2} \xi^{t} H \phi\left(\xi_{0}\right) \xi+O\left(|\xi|^{3}\right),
$$

and then making a linear change of variables for both $x$ and $\xi$ we may further simplify $\frac{1}{2} \xi^{t} H \phi\left(\xi_{0}\right) \xi$ to the form $\frac{1}{2}\left(\xi_{1}^{2} \pm \xi_{2}^{2} \pm \cdots \pm \xi_{d}^{2}\right)=\frac{1}{2} \xi^{t} D \xi$ (diagonalization and rescaling). These operations do not affect the estimate (1.4) except changing the constant $C$. Now one can make the effect of error term small by further scaling

$$
\xi \rightarrow \epsilon_{0} \xi, \quad(x, t) \rightarrow\left(\epsilon_{0}^{-1} x, \epsilon_{0}^{-2} t\right)
$$

which changes $x \cdot \xi+t\left(\frac{1}{2} \xi^{t} D \xi+O\left(|\xi|^{3}\right)\right)$ to $x \cdot \xi+t\left(\frac{1}{2} \xi^{t} D \xi+O\left(\epsilon_{0}|\xi|^{3}\right)\right)$. Hence we get (2.1). 
Asymptotic of oscillatory integral. From the assumption (2.1) $\nabla \phi(\xi)$ is close to $D \xi$. Hence, with a sufficiently small $\epsilon_{0}$ we may assume that $\xi \rightarrow \nabla \phi(\xi)$ is a diffeomorphism on $B(0,2)$ such that there is a unique smooth function $\eta: B(0,15 / 8) \rightarrow \mathbb{R}^{d}$ such that

$$
\nabla \phi(\eta(x))=-x
$$

Then we define

$$
\psi(x)=x \cdot \eta(x)+\phi(\eta(x)) .
$$

Since $\left|\nabla_{\xi}(\xi \cdot x+t \phi(\xi))\right| \gtrsim|x|$ if $|x| \geq 5 t / 4$ (here we are assuming $0<\epsilon_{0} \ll 1$ ), by routine integration by parts we see that for any $M>0$

$$
\left|\int e^{i(x \cdot \xi+t \phi(\xi))} a(\xi) d \xi\right| \lesssim(1+|x|)^{-M}(1+|t|)^{-M}
$$

For the other case $|x|<5 t / 4$ we need the following which can be shown by the stationary phase method. It is a special case of Theorem 7.7.6 in Hörmander [11] (see p.222).

Lemma 2.1. Suppose that $\phi$ is given by (2.1) and $\operatorname{supp} a \subset B(0,1)$. Then, if $t \geq 1$ and $|x| \lesssim t$, for a positive integer $N<L / 2-1$

$$
\int e^{i(x \cdot \xi+t \phi(\xi))} a(\xi) d \xi=\sum_{l=0}^{N} t^{-\frac{d}{2}-l} e^{i t \psi\left(\frac{x}{t}\right)} a_{l}\left(\frac{x}{t}\right)+O\left(|t|^{-N-\frac{d}{2}-1}\right),
$$

where $a_{l}$ is a bounded smooth function with compact support.

\section{Proof of Theorem 1.2}

We first prove the estimate (1.4) and show the weak type endpoint estimate at the end of this section.

Proof of (1.4). To begin with we assume that the operator $E$ is defined by $\phi$ which satisfies (2.1) with a small $\epsilon_{0}>0$ and a large $L$. By time reversal symmetry it is sufficient to show

$$
\|E(f)\|_{L_{t}^{q}\left((0, \infty), L_{x}^{r}\left(\mathbb{R}^{d}\right)\right)} \lesssim\|f\|_{H^{s_{c}}} .
$$

From the Strichartz estimate and Plancherel's theorem we recall the estimate

$$
\|E(f)\|_{L_{t}^{q}\left((0, T), L_{x}^{r}\left(\mathbb{R}^{d}\right)\right)} \lesssim\|f\|_{2}
$$

which holds for $T>0$ and $q, r$ satisfying $d / r+2 / q=d / 2,(r, q, d) \neq(\infty, 2,2)$. By Plancherel's theorem and Hölder's inequality we also have $\|E f\|_{L_{t}^{q}\left((0, T), L_{x}^{2}\left(\mathbb{R}^{d}\right)\right)} \lesssim$ $T^{\frac{1}{q}}\|f\|_{2}$ for $q \geq 1$. Interpolation between these two estimates gives

$$
\|E(f)\|_{L_{t}^{q}\left((0, T), L_{x}^{r}\left(\mathbb{R}^{d}\right)\right)} \lesssim T^{\frac{1}{2}\left(\frac{d}{r}+\frac{2}{q}-\frac{d}{2}\right)}\|f\|_{2}
$$

for $q, r \geq 2$ satisfying $d / r+2 / q \geq d / 2$. Note that $s_{c}>0$ if $d / r+2 / q>d / 2$ and $d / r+1 / q<d / 2$. Hence, we obviously need only to show that

$$
\|E(f)\|_{L_{t}^{q}\left((1, \infty), L_{x}^{r}\left(\mathbb{R}^{d}\right)\right)} \lesssim\|f\|_{H^{s_{c}}} .
$$

From now on we assume that $t \geq 1$. 
Let $\beta \in C_{c}^{\infty}(1 / 2,2)$ such that $\sum_{-\infty}^{\infty} \beta\left(2^{-k} \rho\right)=1$ for $\rho>0$ and let us denote by $P_{k}$ the Littlewood-Paley projection operator which is given by $\mathcal{F}\left(P_{k} f\right)=\beta\left(2^{-k}|\cdot|\right) \widehat{f}$. And we also set $\beta_{0}=1-\sum_{1}^{\infty} \beta\left(2^{-k} \rho\right)$ and define $P_{\leq 0}$ by $\mathcal{F}\left(P_{\leq 0} f\right)=\beta_{0}(|\cdot|) \widehat{f}$.

Using the projection operators, we decompose $E f$ so that

$$
E f=E P_{\leq 0} f+\sum_{k=1}^{\infty} E P_{k} f .
$$

It is easy to handle $E P_{\leq 0} f$. Let us set

$$
K(x, t)=\int e^{i(x \cdot \xi+t \phi(\xi))} a(\xi) d \xi
$$

By Fourier inversion we write

$$
E P_{\leq 0} f(x, t)=\int K(x-y, t) \beta_{0}(|y|) f^{\vee}(y) d y .
$$

Then by (2.3) and Lemma 2.1 $|K(x, t)| \leq t^{-\frac{d}{2}} \chi_{B\left(0, \frac{5}{4}\right)}\left(\frac{x}{t}\right)+(1+|x|)^{-M} t^{-M}$ for $N>0$. Hence it follows that

$$
\left|E P_{\leq 0} f(x, t)\right| \leq C \int\left(t^{-\frac{d}{2}} \chi_{B\left(0, \frac{5}{4}\right)}\left(\frac{x-y}{t}\right)+(1+|x-y|)^{-M} t^{-M}\right)\left|\beta_{0}(|y|) f^{\vee}(y)\right| d y .
$$

Since $\beta_{0}(|\cdot|)$ is supported in $B(0,2)$, by Cauchy-Schwarz inequality and Plancherel's theorem we see that

$$
\begin{aligned}
\left|E P_{\leq 0} f(x, t)\right| & \leq C t^{-\frac{d}{2}}\left(\chi_{\left\{|x| \leq \frac{5}{4} t+2\right\}}+(1+|x|)^{-M}\right)\left\|\beta_{0}(|\cdot|) f^{\vee}\right\|_{1} \\
& \leq C t^{-\frac{d}{2}}\left(\chi_{\left\{|x| \leq \frac{5}{4} t+2\right\}}+(1+|x|)^{-M}\right)\|f\|_{2} .
\end{aligned}
$$

So, by taking integration it follows that

$$
\left\|E P_{\leq 0} f\right\|_{L_{t}^{q}\left((1, \infty), L_{x}^{r}\left(\mathbb{R}^{d}\right)\right)} \leq C\left\|t^{-\frac{d}{2}+\frac{d}{r}}\right\|_{L_{t}^{q}(1, \infty)}\|f\|_{2} \leq C\|f\|_{2}
$$

because $d / r+1 / q<d / 2$.

For $k \geq 1$ and a large constant $C>0$, we set

$$
\chi_{k}^{\circ}(t)=\chi_{\left[1, C 2^{k}\right]}(t), \quad \chi_{k}^{c}(t)=\chi_{\left[C 2^{k}, \infty\right)}(t) .
$$

We break the sum in (3.2) so that

$$
\sum_{k=1}^{\infty} E P_{k} f=\sum_{k=1}^{\infty} \chi_{k}^{\circ} E P_{k} f+\sum_{k=1}^{\infty} \chi_{k}^{c} E P_{k} f .
$$

The contribution from the first summation is rather easy to handle. In fact, since $\frac{d}{r}+\frac{2}{q}-\frac{d}{2}>0$, using $(3.1)$

$$
\begin{aligned}
& \left\|\sum_{k=1}^{\infty} \chi_{k}^{\circ} E P_{k} f\right\|_{L_{t}^{q} L_{x}^{r}} \leq \sum_{k=1}^{\infty}\left\|\chi_{k}^{\circ} E P_{k} f\right\|_{L_{t}^{q} L_{x}^{r}} \leq C \sum_{k=1}^{\infty} 2^{\frac{k}{2}\left(\frac{d}{r}+\frac{2}{q}-\frac{d}{2}\right)}\left\|P_{k} f\right\|_{L^{2}} \\
& \leq C\left(\sum_{k=1}^{\infty} 2^{-k\left(\frac{d}{r}+\frac{2}{q}-\frac{d}{2}\right)}\right)^{\frac{1}{2}}\left(\sum_{k=1}^{\infty} 2^{2 k\left(\frac{d}{r}+\frac{2}{q}-\frac{d}{2}\right)}\left\|P_{k} f\right\|_{L^{2}}^{2}\right)^{\frac{1}{2}} \leq C\|f\|_{H^{s_{c}}}
\end{aligned}
$$


Hence we are reduced to showing

$$
\left\|\sum_{k=1}^{\infty} \chi_{k}^{c} E P_{k} f\right\|_{L_{t}^{q} L_{x}^{r}} \leq C\|f\|_{H^{s_{c}}} .
$$

We now use the asymptotic expansion in Lemma 2.1. Let $A$ be a smooth function supported in $[-3 / 2,3 / 2]$ such that $A=1$ on $[-5 / 4,5 / 4]$. Then we use Lemma 2.1 for $K(x, t) A\left(\frac{|x|}{t}\right)$ and (2.3) for $K(x, t)\left(1-A\left(\frac{|x|}{t}\right)\right)$ to get

$$
K(x, t)=\sum_{l=0}^{N} t^{-\frac{d}{2}-l} e^{i t \psi\left(\frac{x}{t}\right)} A_{l}\left(\frac{x}{t}\right)+e(x, t),
$$

where $e(x, t)=O\left((1+|x|+t)^{-N-\frac{d}{2}-1}\right)$ and $A_{l}$ is a smooth function supported in $B(0,3 / 2)$. For simplicity we set

$$
\widetilde{A}\left(\frac{x}{t}\right)=\sum_{l=0}^{N} t^{-l} A_{l}\left(\frac{x}{t}\right) .
$$

We now define

$$
\widetilde{E} f(x, t)=t^{-\frac{d}{2}} \int e^{i t \psi\left(\frac{x-y}{t}\right)} \widetilde{A}\left(\frac{x-y}{t}\right) f^{\vee}(y) d y, \quad \mathcal{R} f(x, t)=\int e(x-y, t) f^{\vee}(y) d y .
$$

Since $E f=\int K(x-y, t) f^{\vee}(y) d y$, clearly $E f=\widetilde{E} f+\mathcal{R} f$. The left hand side of (3.4) is bounded by

$$
\left\|\sum_{k=1}^{\infty} \chi_{k}^{c} \widetilde{E} P_{k} f\right\|_{L_{t}^{q} L_{x}^{r}}+\left\|\sum_{k=1}^{\infty} \chi_{k}^{c} \mathcal{R} P_{k} f\right\|_{L_{t}^{q} L_{x}^{r}} .
$$

The contribution from $\sum_{k=1}^{\infty} \chi_{k}^{c} \mathcal{R} P_{k} f$ is easy to control. In fact, since $q, r \geq 2$, with a sufficiently large $N$ (using $e(x, t)=O\left((1+|x|+t)^{-N-1}\right)$ ) and by Young's inequality we see that for $s>0$

$$
\begin{aligned}
& \left\|\sum_{k=1}^{\infty} \chi_{k}^{c} \mathcal{R} P_{k} f\right\|_{L_{t}^{q} L_{x}^{r}} \leq \sum_{k=1}^{\infty}\left\|\chi_{k}^{c} \mathcal{R} P_{k} f\right\|_{L_{t}^{q} L_{x}^{r}} \\
\leq C & \sum_{k=1}^{\infty}\left\|t^{-1}\right\| \int(1+|\cdot-y|)^{-N}\left|\beta\left(2^{-k}|y|\right) f^{\vee}(y)\right| d y\left\|_{L_{x}^{r}}\right\|_{L_{t}^{q}(1, \infty)} \\
\leq & C \sum_{k=1}^{\infty}\left\|\beta\left(2^{-k}|\cdot|\right) f^{\vee}\right\|_{2} \leq C\|f\|_{H^{s}} .
\end{aligned}
$$

For the last inequality we use the Cauchy-Schwarz inequality and Plancherel's theorem. To get the desired bound, by multiplying harmless factor $e^{-i t \psi(x / t)}$ it is sufficient to consider the operator $\widetilde{E_{\psi}}$ which is defined by

$$
\widetilde{E_{\psi}} f(x, t)=e^{-i t \psi\left(\frac{x}{t}\right)} \widetilde{E} f(x, t) .
$$

The estimate (3.4) follows from

$$
\left\|\sum_{k=1}^{\infty} \chi_{k}^{c}(t) \widetilde{E_{\psi}} P_{k} f\right\|_{L_{t}^{q} L_{x}^{r}} \leq C\|f\|_{H^{s_{c}}} .
$$


Let us set

$$
m(k, y, \xi)=2^{k d} \int e^{i\left(t \psi\left(x-\frac{2^{k} y}{t}\right)-t \psi(x)-x \cdot \xi\right)} \widetilde{A}\left(x-\frac{2^{k} y}{t}\right) d x .
$$

Then by scaling $y \rightarrow 2^{k} y$ we get

$$
\mathcal{F}_{x}\left(\widetilde{E_{\psi}} P_{k} f(t \cdot, t)\right)(\xi)=t^{-\frac{d}{2}} \int m(k, y, \xi) \beta(|y|) f^{\vee}\left(2^{k} y\right) d y
$$

Here $\mathcal{F}_{x}$ denotes the Fourier transform in $x$. In order to get (3.5) we need the following lemma which shows that if $t \gg 2^{k}$, the Fourier transform of $\widetilde{E_{\psi}} P_{k} f(t \cdot, t)$ is essentially supported in the set $\left\{\xi:|\xi| \sim 2^{k}\right\}$.

Lemma 3.1. Let $1 / 2 \leq|y| \leq 2$. If $|\xi| \geq B 2^{k}$ or $|\xi| \leq B^{-1} 2^{k}$ for some large $B>0$, then for any $M>0$ and multi-index $\alpha$

$$
\left|\partial_{\xi}^{\alpha} m(k, y, \xi)\right| \leq C\left(\max \left\{2^{k},|\xi|\right\}\right)^{-M}
$$

with $C$ independent of $k, y$.

Proof. To see this, we consider the phase function of the integral in (3.6)

$$
t \psi\left(x-\frac{2^{k} y}{t}\right)-t \psi(x)-x \cdot \xi
$$

From (2.2) and (2.1) we have $\nabla \psi(x)=\eta(x)=D x+\mathcal{E}(x)$ where $\|\mathcal{E}\|_{C^{L}(B(0,2))} \lesssim \epsilon_{0}$. The Hessian matrix of $\psi$ is close to the matrix $D$. Since $|y| \sim 1$ and $\frac{2^{k}}{t} \ll 1$, it is easy to see

$$
\left|\nabla \psi\left(x-\frac{2^{k} y}{t}\right)-\nabla \psi(x)\right|=\left|M \frac{2^{k} y}{t}+O\left(\epsilon_{0} \frac{2^{k} y}{t}\right)\right| \sim \frac{2^{k}}{t} .
$$

Since $|\xi| \geq B 2^{k}$ or $|\xi| \leq B^{-1} 2^{k}$ for some large $B>0$, we get

$$
\left|\nabla_{x}\left(t \psi\left(x-\frac{2^{k} y}{t}\right)-t \psi(x)-x \cdot \xi\right)\right| \gtrsim \max \left(2^{k},|\xi|\right) .
$$

Note that $\widetilde{A}\left(\cdot-\frac{2^{k} y}{t}\right)$ is supported in $B(0,7 / 4)$. By integration by part we get the desired inequality.

Now we break

$$
\widetilde{E_{\psi}} P_{k} f(t \cdot, t)=\left(I-\widetilde{P}_{k}\right) \widetilde{E_{\psi}} P_{k} f(t \cdot, t)+\widetilde{P}_{k} \widetilde{E_{\psi}} P_{k} f(t \cdot, t),
$$

where $\widetilde{P}_{k}$ is a projection operator defined by $\mathcal{F}\left(\widetilde{P}_{k} f\right)=\widetilde{\beta}\left(2^{-k}|\xi|\right) \widehat{f}(\xi)$ with $\widetilde{\beta} \in$ $\left.C_{0}^{\infty}(1 / 2 B, 2 B)\right)$ satisfying $\widetilde{\beta}=1$ on $\left(B^{-1}, B\right)$. By integration by parts with (3.8), it follows that if $1 / 2 \leq|y| \leq 2$

$$
\left|\mathcal{F}^{-1}\left(\left(1-\widetilde{\beta}\left(2^{-k}|\cdot|\right)\right) m(k, y, \cdot)\right)\right| \leq C 2^{-M k}(1+|x|)^{-M}
$$

for any $M$. Hence, by (3.7) we get

$$
\left|\left(I-\widetilde{P}_{k}\right) \widetilde{E_{\psi}} P_{k} f(t x, t)\right| \leq C t^{-\frac{d}{2}} 2^{-M k}(1+|x|)^{-M} \int\left|\beta(|y|) f^{\vee}\left(2^{k} y\right)\right| d y .
$$


Then, by Schwarz's inequality and Plancherel's theorem, $\left|\left(I-\widetilde{P}_{k}\right) \widetilde{E_{\psi}} P_{k} f(t x, t)\right| \leq$ $C t^{-\frac{d}{2}} 2^{-M k}(1+|x|)^{-M}\left\|P_{k} f\right\|_{2}$ and thus

$$
\left\|\chi_{k}^{c}(t)\left(I-\widetilde{P}_{k}\right) \widetilde{E_{\psi}} P_{k} f(t \cdot, t)\right\|_{L_{x}^{r}} \leq C t^{-\frac{d}{2}} 2^{-M k}\left\|P_{k} f\right\|_{2} .
$$

Using this and Littlewood-Paley inequality, we see that

$$
\begin{aligned}
& \left\|\sum_{k=1}^{\infty} \chi_{k}^{c} \widetilde{E_{\psi}} P_{k} f(\cdot, t)\right\|_{L_{x}^{r}}=t^{\frac{d}{r}}\left\|\sum_{k=1}^{\infty} \chi_{k}^{c} \widetilde{E_{\psi}} P_{k} f(t \cdot, t)\right\|_{L_{x}^{r}} \\
\leq & t^{\frac{d}{r}}\left\|\sum_{k=1}^{\infty} \chi_{k}^{c} \widetilde{P}_{k} \widetilde{E_{\psi}} P_{k} f(t \cdot, t)\right\|_{L_{x}^{r}}+t^{\frac{d}{r}}\left\|\sum_{k=1}^{\infty} \chi_{k}^{c}\left(I-\widetilde{P}_{k}\right) \widetilde{E_{\psi}} P_{k} f(t \cdot, t)\right\|_{L_{x}^{r}} \\
\leq & C t^{\frac{d}{r}} \chi_{k}^{c}(t)\left\|\left(\sum_{k=1}^{\infty}\left|\widetilde{E_{\psi}} P_{k} f(t \cdot, t)\right|^{2}\right)^{\frac{1}{2}}\right\|_{L_{x}^{r}}+C t^{\frac{d}{r}-\frac{d}{2}} \sum_{k=1}^{\infty} 2^{-M k}\|f\|_{2} .
\end{aligned}
$$

Since $q, r \geq 2$, taking integration in $t$, by Minkowski's inequality we get

$$
\begin{gathered}
\left\|\sum_{k=1}^{\infty} \chi_{k}^{c} \widetilde{E_{\psi}} P_{k} f(\cdot, t)\right\|_{L_{t}^{q} L_{x}^{r}} \leq C\left(\sum_{k=1}^{\infty}\left\|t^{\frac{d}{r}} \chi_{k}^{c}\right\| \widetilde{E_{\psi}} P_{k} f(t \cdot, t)\left\|_{L_{x}^{r}}\right\|_{L_{t}^{q}}^{2}\right)^{\frac{1}{2}} \\
+\sum_{k=1}^{\infty} 2^{-M k}\left\|P_{k} f\right\|_{2}\left\|t^{\frac{d}{r}-\frac{d}{2}}\right\|_{q} .
\end{gathered}
$$

The second term in the right hand side is clearly bounded by $C\|f\|_{H^{s}}$ if $d / r+1 / q<$ $d / 2$. Therefore we are reduced to showing that

$$
\left(\sum_{k=1}^{\infty}\left\|t^{\frac{d}{r}} \chi_{k}^{c}\right\| \widetilde{E_{\psi}} P_{k} f(t \cdot, t)\left\|_{L_{x}^{r}}\right\|_{L_{t}^{q}}^{2}\right)^{\frac{1}{2}} \leq C\|f\|_{H^{s}} .
$$

For this we use the following.

Lemma 3.2. If $t \geq B 2^{2 k}$ for some large $B>0$, then for $1 \leq r \leq \infty$

$$
\left\|\widetilde{E_{\psi}} P_{k} f(t \cdot, t)\right\|_{L_{x}^{r}} \leq C t^{-\frac{d}{2}}\left\|P_{k} f\right\|_{r}
$$

Proof. Since $\left(P_{k} f\right)^{\vee}$ is supported in $\left\{y: 2^{k-1} \leq|y| \leq 2^{k+1}\right\}$, we may insert a harmless smooth function $\beta_{0}$ so that

$$
\widetilde{E_{\psi}} P_{k} f(x, t)=e^{-i t \psi\left(\frac{x}{t}\right)} t^{-\frac{d}{2}} \int e^{i t \psi\left(\frac{x-y}{t}\right)} \widetilde{A}\left(\frac{x-y}{t}\right) \beta_{\circ}\left(2^{-k}|y|\right)\left(P_{k} f\right)^{\vee}(y) d y,
$$

where $\beta=\beta_{\circ} \beta$ and $\beta_{\circ}$ is supported in $\left[2^{-2}, 2^{2}\right]$. By rescaling we have

$$
\widetilde{E_{\psi}} P_{k} f(t x, t)=t^{-\frac{d}{2}} \int \mathcal{K}(x, z, k) P_{k} f(z) d z,
$$

where

$$
\mathcal{K}(x, z, k)=(2 \pi)^{-d} 2^{d k} e^{-i t \psi(x)} \int e^{i t \psi\left(x-\frac{2^{k} y}{t}\right)} e^{i 2^{k} z \cdot y} \widetilde{A}\left(x-\frac{2^{k} y}{t}\right) \beta_{\circ}(|y|) d y .
$$

Since $|y| \sim 1$ and $t \geq B 2^{2 k}$, considering the phase part of this integral, we see that $\nabla_{y}\left(t \psi\left(x-\frac{2^{k} y}{t}\right)+2^{k} z y\right)=2^{k}(z-\nabla \psi(x))+O(1)$. Therefore, by integration by parts we get

$$
|\mathcal{K}(x, z, k)| \leq C 2^{k d}\left(1+2^{k}|z-\nabla \psi(x)|\right)^{-N} .
$$


Since $\widetilde{A}$ is supported in $B(0,3 / 2)$, it follows that $\operatorname{supp} \mathcal{K}(\cdot, z, k) \subset B(0,7 / 4)$ if $B$ is sufficiently large. From (2.2) $x \rightarrow \nabla \psi(x)$ is a diffeomorphism on $B(0,15 / 8)$. Hence it is easy to see $\int|\mathcal{K}(x, z, k)| d x<C$. Clearly, $\int|\mathcal{K}(x, z, k)| d z \leq C$. Then, (3.11) follows from Young's inequality.

We now return to the proof of (3.10). We break $\chi_{k}^{c}$ to $\chi_{\left[C 2^{k}, C 2^{2 k}\right]}+\chi_{\left[C 2^{2 k}, \infty\right)}$ so that

$$
\begin{gathered}
\left(\sum_{k=1}^{\infty}\left\|t^{\frac{d}{r}} \chi_{k}^{c}\right\| \widetilde{E_{\psi}} P_{k} f(t \cdot, t)\left\|L_{x}^{r}\right\|_{L_{t}^{q}}^{2}\right)^{\frac{1}{2}} \leq\left(\sum_{k=1}^{\infty}\left\|t^{\frac{d}{r}} \chi_{\left[C 2^{k}, C 2^{2 k}\right]}(t)\right\| \widetilde{E_{\psi}} P_{k} f(t \cdot, t)\left\|_{L_{x}^{r}}\right\|_{L_{t}^{q}}^{2}\right)^{\frac{1}{2}} \\
+\left(\sum_{k=1}^{\infty}\left\|t^{\frac{d}{r}} \chi_{\left[C 2^{2 k}, \infty\right)}\right\| \widetilde{E_{\psi}} P_{k} f(t \cdot, t)\left\|_{L_{x}^{r}}\right\|_{L_{t}^{q}}^{2}\right)^{\frac{1}{2}} .
\end{gathered}
$$

By rescaling we note that the first term of right hand side equals

$$
\left(\sum_{k=1}^{\infty}\left\|\widetilde{E_{\psi}} P_{k} f\right\|_{L_{t}^{q}\left(\left[C 2^{k}, C 2^{2 k}\right], L_{x}^{r}\left(\mathbb{R}^{d}\right)\right)}^{2}\right)^{\frac{1}{2}} .
$$

By (3.1) it follows that this is bounded by $C\left(\sum_{k=1}^{\infty} 2^{2 k\left(\frac{d}{r}+\frac{2}{q}-\frac{d}{2}\right)}\left\|P_{k} f\right\|_{2}^{2}\right)^{\frac{1}{2}} \lesssim\|f\|_{H^{s_{c}}}$. So, we only need to consider the second term. By making use of (3.11) we see

$$
\begin{aligned}
& \left(\sum_{k=1}^{\infty}\left\|t^{\frac{d}{r}} \chi_{\left[C 2^{2 k}, \infty\right)}\right\| \widetilde{E_{\psi}} P_{k} f(t \cdot, t)\left\|_{L_{x}^{r}}\right\|_{L_{t}^{q}}^{2}\right)^{\frac{1}{2}} \leq C\left(\sum_{k=1}^{\infty}\left\|t^{\frac{d}{r}} t^{-\frac{d}{2}} \chi_{\left[C 2^{2 k}, \infty\right)}\right\|_{L_{t}^{q}}^{2}\left\|P_{k} f\right\|_{r}^{2}\right)^{\frac{1}{2}} \\
& \leq C\left(\sum_{k=1}^{\infty} 2^{4 k\left(\frac{d}{r}+\frac{1}{q}-\frac{d}{2}\right)}\left\|P_{k} f\right\|_{r}^{2}\right)^{\frac{1}{2}} \leq C\left(\sum_{k=1}^{\infty} 2^{2 k\left(\frac{d}{r}+\frac{2}{q}-\frac{d}{2}\right)}\left\|P_{k} f\right\|_{2}^{2}\right)^{\frac{1}{2}} \leq C\|f\|_{H^{s_{c}}} .
\end{aligned}
$$

For the last inequality we use Bernstein's inequality $\left\|P_{k} f\right\|_{q} \leq C 2^{k\left(\frac{d}{2}-\frac{d}{q}\right)}\left\|P_{k} f\right\|_{2}$. This completes the proof of (3.10).

Proof of weak type endpoint estimate. Let us fix $q, r$ such that $2 \leq q, r<\infty$ and $d / r+1 / q=d / 2$. The proof here is a minor modification of that of (1.4). So we shall be brief.

As before it suffices to show $\|E(f)\|_{L_{t}^{q, \infty}\left((0, \infty), L_{x}^{r}\left(\mathbb{R}^{d}\right)\right)} \lesssim\|f\|_{H^{s_{c}}}$. Because of (3.1) it is enough to prove that

$$
\|E(f)\|_{L_{t}^{q, \infty}\left((1, \infty), L_{x}^{r}\left(\mathbb{R}^{d}\right)\right)} \lesssim\|f\|_{H^{s_{c}}}
$$

By (3.3) it follows that

$$
\left\|E P_{\leq 0} f\right\|_{L_{t}^{q, \infty}\left((1, \infty), L_{x}^{r}\left(\mathbb{R}^{d}\right)\right)} \leq C\left\|t^{-\frac{d}{2}+\frac{d}{r}}\right\|_{L_{t}^{q, \infty}(1, \infty)}\|f\|_{2} \leq C\|f\|_{2}
$$

because $t^{-\frac{d}{2}+\frac{d}{r}}=t^{-\frac{1}{q}} \in L^{q, \infty}(0, \infty)$. Breaking $\sum_{k=1}^{\infty} E P_{k} f=\sum_{k=1}^{\infty} \chi_{k}^{\circ} E P_{k} f+$ $\sum_{k=1}^{\infty} \chi_{k}^{c} E P_{k} f$, for the first sum we get the desired bound by the same argument as before because $L^{q} \subset L^{q, \infty}$. Hence, it is enough to show

$$
\left\|\sum_{k=1}^{\infty} \chi_{k}^{c} E P_{k} f\right\|_{L_{t}^{q, \infty}\left((1, \infty), L_{x}^{r}\left(\mathbb{R}^{d}\right)\right)} \leq C\|f\|_{H^{s_{c}}} .
$$


Decomposing further $\chi_{k}^{c} E P_{k} f=\chi_{k}^{c} \widetilde{E} P_{k} f+\chi_{k}^{c} \mathcal{R} P_{k} f$, the contribution from $\chi_{k}^{c} \mathcal{R} P_{k} f$ is controlled by the bound obtained previously. So it is sufficient to show that

$$
\left\|\sum_{k=1}^{\infty} \chi_{k}^{c} \widetilde{E_{\psi}} P_{k} f\right\|_{L_{t}^{q, \infty}\left((1, \infty), L_{x}^{r}\left(\mathbb{R}^{d}\right)\right)} \leq C\|f\|_{H^{s_{c}}} .
$$

Since $q>2, L^{q / 2, \infty}$ is a Banach space. Using Minkowski's and triangle inequalities, from (3.9) we get

$$
\begin{gathered}
\left\|\sum_{k=1}^{\infty} \chi_{k}^{c} \widetilde{E_{\psi}} P_{k} f(\cdot, t)\right\|_{L_{t}^{q, \infty}\left((1, \infty), L_{x}^{r}\left(\mathbb{R}^{d}\right)\right)} \leq C\left(\sum_{k=1}^{\infty}\left\|t^{\frac{d}{r}} \chi_{k}^{c}\right\| \widetilde{E_{\psi}} P_{k} f(t \cdot, t)\left\|_{L_{x}^{r}}\right\|_{L_{t}^{q, \infty}}^{2}\right)^{\frac{1}{2}} \\
+\sum_{k=1}^{\infty} 2^{-N k}\left\|P_{k} f\right\|_{2}\left\|t^{\frac{d}{r}-\frac{d}{2}}\right\|_{L^{q, \infty}}
\end{gathered}
$$

Since $t^{\frac{d}{r}-\frac{d}{2}} \in L^{q, \infty}(0, \infty)$, the second term on the right hand side is bounded by $C\|f\|_{H^{s_{c}}}$ and

$$
\left\|t^{\frac{d}{r}} \chi_{\left[C 2^{2 k}, \infty\right)}\right\| \widetilde{E_{\psi}} P_{k} f(t \cdot, t)\left\|_{L_{x}^{r}}\right\|_{L_{t}^{q, \infty}} \leq C 2^{k\left(\frac{d}{r}+\frac{2}{q}-\frac{d}{2}\right)}\left\|P_{k} f\right\|_{2} .
$$

As before this follows from (3.11) and Bernstein's inequality. This completes the proof.

\section{Strichartz estimates: Proofs of Corollaries}

In this section we prove Corollary 1.3, 1.4 and 1.5,

Proof of Corollary 1.3. Since $q, r \geq 2$, using Littlewood-Paley theory and Minkowski's inequality, we have

$$
\left\|e^{i t(-\Delta)^{\alpha / 2}} \varphi\right\|_{L_{t}^{q}\left(\mathbb{R}, L_{x}^{r}\left(\mathbb{R}^{d}\right)\right)}^{2} \lesssim \sum_{k \in \mathbb{Z}}\left\|e^{i t(-\Delta)^{\alpha / 2}} P_{k} \varphi\right\|_{L_{t}^{q}\left(\mathbb{R}, L_{x}^{r}\left(\mathbb{R}^{d}\right)\right)}^{2} .
$$

We observe that $e^{i t(-\Delta)^{\alpha / 2}} P_{k} g(x)=2^{d k} e^{i t 2^{\alpha k}(-\Delta)^{\alpha / 2}} P_{0} g_{2^{k}}\left(2^{k} x\right)$, where $g_{\lambda}(x)=\lambda^{-d} g(x / \lambda)$. Since $0<s_{c}<1 / 2$, recalling Remark1, from rescaling and Theorem 1.2 we get

$$
\begin{aligned}
\left\|e^{i t(-\Delta)^{\alpha / 2}} \varphi\right\|_{L_{t}^{q}\left(\mathbb{R}, L_{x}^{r}\left(\mathbb{R}^{d}\right)\right)}^{2} & \lesssim \sum_{k \in \mathbb{Z}} 2^{2 k\left(d-\frac{d}{r}-\frac{\alpha}{q}\right)}\left\|e^{i t(-\Delta)^{\alpha / 2}} P_{0} \varphi_{2^{k}}\right\|_{L_{t}^{q}\left(\mathbb{R}, L_{x}^{r}\left(\mathbb{R}^{d}\right)\right)}^{2} \\
& \lesssim \sum_{k \in \mathbb{Z}} 2^{2 k\left(d-\frac{d}{r}-\frac{\alpha}{q}\right)}\left\|\beta \widehat{\varphi}\left(2^{k} \cdot\right)\right\|_{\dot{H}^{s_{c}}}^{2}
\end{aligned}
$$

By Plancherel's theorem and rescaling (note that $P_{0} \varphi_{2^{k}}=2^{-k d}\left(P_{k} \varphi\right)\left(2^{-k} x\right)$ ) it follows that

$$
\begin{aligned}
\left\|e^{i t(-\Delta)^{\alpha / 2}} \varphi\right\|_{L_{t}^{q}\left(\mathbb{R}, L_{x}^{r}\left(\mathbb{R}^{d}\right)\right)}^{2} & \lesssim \sum_{k \in \mathbb{Z}} 2^{2 k\left(d-\frac{d}{r}-\frac{\alpha}{q}\right)}\left\||x|^{s_{c}} P_{0} \varphi_{2^{k}}\right\|_{2}^{2} \\
& \lesssim \sum_{k \in \mathbb{Z}} 2^{2 k(2-\alpha) / q}\left\||x|^{s_{c}} P_{k} \varphi\right\|_{2}^{2}
\end{aligned}
$$


We define $\mathcal{P}_{k}$ by $\mathcal{F}\left(\mathcal{P}_{k} f\right)=\beta_{\circ}\left(2^{-k}|\cdot|\right) \widehat{f}$ so that $\mathcal{P}_{k} P_{k}=P_{k}$. (Here $\beta_{\circ}$ is a smooth function supported in $\left[2^{-2}, 2^{2}\right]$ such that $\beta_{\circ} \beta=\beta$.) Also we set $\widetilde{P}_{k}=$ $2^{k \frac{(2-\alpha)}{q}}(-\Delta)^{\frac{\alpha-2}{2 q}} \mathcal{P}_{k}$. Hence we get

$$
\left\|e^{i t(-\Delta)^{\alpha / 2}} \varphi\right\|_{L_{t}^{q}\left(\mathbb{R}, L_{x}^{r}\left(\mathbb{R}^{d}\right)\right)}^{2} \lesssim \int\left(\sum_{k \in \mathbb{Z}}\left|\widetilde{P}_{k} P_{k}(-\Delta)^{\frac{2-\alpha}{2 q}} \varphi\right|^{2}\right)|x|^{2 s_{c}} d x .
$$

Since $0<s_{c}<1 / 2,|x|^{2 s_{c}}$ is an $A_{2}$-weight (see [18, p.219]). Thus by a vector valued inequality for $A_{p}$ weight (e.g. [8, Remarks 6.5, p. 521]) it follows that

$$
\left\|e^{i t(-\Delta)^{\alpha / 2}} \varphi\right\|_{L_{t}^{q}\left(\mathbb{R}, L_{x}^{r}\left(\mathbb{R}^{d}\right)\right)}^{2} \lesssim \int\left(\sum_{k \in \mathbb{Z}}\left|P_{k}(-\Delta)^{\frac{2-\alpha}{2 q}} \varphi\right|^{2}\right)|x|^{2 s_{c}} d x .
$$

By Littlewood-Paley theory (e.g. [16, p. 275], [25]) in weighted $L^{p}$ spaces the right hand side is bounded by

$$
C \int\left|(-\Delta)^{\frac{2-\alpha}{2 q}} \varphi\right|^{2}|x|^{2 s_{c}} d x
$$

Therefore we get the desired inequality.

Proof of Corollary 1.4. In order to prove Corollary 1.4, it is sufficient to show that

$$
\left\|e^{i t \sqrt{-\Delta}} \varphi\right\|_{L_{t}^{q}\left(\mathbb{R}, L_{x}^{r}\left(\mathbb{R}^{d}\right)\right)} \leq C\left\||x|^{s_{c}^{w}} \varphi\right\|_{2}
$$

whenever $\widehat{\varphi}$ is supported in $\{\xi: 1 / 2<|\xi|<2\}$. Once it is established, the rest of proof is identical with that of Corollary 1.3. By a finite decomposition, rotation and rescaling we may assume that $\widehat{f}$ is supported in $\Gamma=\left\{\xi=\left(\bar{\xi}, \xi_{d}\right):|\bar{\xi}|<\right.$ $\left.\xi_{d} / 100,1 / 2<\xi_{d}<2\right\}$. Let us set

$$
\mathcal{T} \varphi(x, t)=\int_{\Gamma} e^{i x \cdot \xi+i t\left(|\xi|-\xi_{d}\right)} \widehat{\varphi}(\xi) d \xi=\int_{\Gamma} e^{i \bar{x} \cdot \bar{\xi}+i x_{d} \xi_{d}+i t \xi_{d} \theta\left(\bar{\xi} / \xi_{d}\right)} \widehat{\varphi}(\xi) d \xi
$$

with $\theta(\eta)=\sqrt{1+|\eta|^{2}}-1$. Then, by a simple change of variables $x_{d} \rightarrow x_{d}-t$ it is enough to show that

$$
\|\mathcal{T} \varphi\|_{L_{t}^{q}\left(\mathbb{R}, L_{x}^{r}\left(\mathbb{R}^{d}\right)\right)} \leq C\left\||\bar{x}|^{s_{c}^{w}} \varphi\right\|_{2}
$$

provided that supp $\widehat{\varphi} \subset \Gamma$. By the Hausdorff-Young inequality in $x_{d}$ and Minkowski's inequality, the left hand side is bounded by

$$
C\left(\int_{1 / 2}^{2}\left\|\int_{|\bar{\xi}| \leq \frac{1}{50}} e^{i \bar{x} \cdot \bar{\xi}+i t \xi_{d} \theta\left(\bar{\xi} / \xi_{d}\right)} \widehat{\varphi}\left(\bar{\xi}, \xi_{d}\right) d \bar{\xi}\right\|_{L_{t}^{q}\left(\mathbb{R}, L_{\bar{x}}^{r}\left(\mathbb{R}^{d-1}\right)\right)}^{r^{\prime}} d \xi_{d}\right)^{\frac{1}{r^{\prime}}} .
$$

Freezing $\xi_{d} \in(1 / 2,2)$, the Hessian matrix of $\theta\left(\cdot / \xi_{d}\right)$ is non singular. So, we apply Theorem 1.2 to the extension operator defined by $\theta\left(\cdot / \xi_{d}\right)$. In fact, since $\theta(\eta)$ is close to $\frac{1}{2}|\eta|^{2}$ and $1 / 2 \leq \xi_{d} \leq 2$, it is easy to see that there is a uniform bound $C$ independent of $\xi_{d}$ so that

$$
\left\|\int_{|\bar{\xi}| \leq \frac{1}{50}} e^{i \bar{x} \cdot \bar{\xi}+i t \xi_{d} \theta\left(\bar{\xi} / \xi_{d}\right)} g(\bar{\xi}) d \bar{\xi}\right\|_{L_{t}^{q}\left(\mathbb{R}, L_{\bar{x}}^{r}\left(\mathbb{R}^{d-1}\right)\right)} \leq C\left\|\left(1-\Delta_{\bar{x}}\right)^{s_{c}^{w} / 2} g\right\|_{2} .
$$

Therefore, recalling Remark 1 and taking integration in $\xi_{d}$, we get

$$
\|\mathcal{T} \varphi\|_{L_{t}^{q}\left(\mathbb{R}, L_{x}^{r}\left(\mathbb{R}^{d}\right)\right)} \leq C\left(\int_{1 / 2}^{2}\left\|\left(-\Delta_{\bar{x}}\right)^{s_{c}^{w} / 2} \widehat{\varphi}\left(\bar{\xi}, \xi_{d}\right)\right\|_{L^{2}}^{r^{\prime}} d \xi_{d}\right)^{\frac{1}{r^{\prime}}} .
$$


Then (4.1) follows by Plancherel's theorem and Hölder's inequality.

Proof of Corollary 1.5. For the proof it suffices to show the case $q=r$. The other cases follow from interpolation with the estimate $\left\|(-\Delta)^{\gamma_{1}(\infty, 2, d) / 2} e^{i t \sqrt{-\Delta}} \varphi\right\|_{L_{t}^{\infty}\left(\mathbb{R}, L_{x}^{2}\left(\mathbb{R}^{d}\right)\right)}$ $\lesssim C\|\varphi\|_{H_{s p h}^{0}}$. By Littlewood-Paley theory it is enough to show that

$$
\left\|e^{i t \sqrt{-\Delta}} \varphi\right\|_{L_{t}^{q}\left(\mathbb{R}, L_{x}^{r}\left(\mathbb{R}^{d}\right)\right)} \lesssim C\|\varphi\|_{H_{s p h}^{s_{c}^{w}}}
$$

for $\varphi$ of which Fourier transform is supported in $\{\xi: 1 / 2 \leq|\xi| \leq 2\}$. For this we write

$$
e^{i t \sqrt{-\Delta}} \varphi(x)=\frac{1}{(2 \pi)^{d}} \int_{\frac{1}{2}}^{2} \int_{\mathbb{S}^{d-1}} e^{i \rho x \cdot \omega+i t \rho} \widehat{\varphi}(\rho \omega) d \omega \rho^{d-1} d \rho .
$$

By the Hausdorff-Young inequality in $t$ and Minkowski's inequality we get

$$
\left\|e^{i t \sqrt{-\Delta}} \varphi\right\|_{L_{x}^{q}\left(\mathbb{R}^{d}, L_{t}^{q}(\mathbb{R})\right)} \lesssim\left\|R^{*}(\widehat{\varphi}(\rho \cdot))(\rho \cdot)\right\|_{L_{\rho}^{q^{\prime}}\left((1 / 2,2): L_{x}^{q}\left(\mathbb{R}^{d}\right)\right)} .
$$

Theorem 1.1 and Hölder's inequality imply

$$
\left.\left\|e^{i t \sqrt{-\Delta}} \varphi\right\|_{L_{x}^{q}\left(\mathbb{R}^{d}, L_{t}^{q}(\mathbb{R})\right)} \lesssim \|\left(1-\Delta_{\omega}\right)^{s_{c}^{w} / 2} \widehat{\varphi}(\rho \omega)\right) \|_{L_{\rho}^{2}\left((1 / 2,2): L_{\omega}^{2}\left(\mathbb{S}^{d-1}\right)\right)} .
$$

Now recalling $\left(1-\Delta_{\omega}\right)^{\nu / 2} \widehat{g}=\mathcal{F}\left(\left(1-\Delta_{\omega}\right)^{\nu / 2} g\right)$, we get the desired inequality by Plancherel's theorem.

Acknowledgment. Y. Cho is supported by NRF grant 2011-0005122 (Republic of Korea). Z. Guo is supported in part by NNSF of China (No.11371037), Beijing Higher Education Young Elite Teacher Project (No. YETP0002), and Fok Ying Tong education foundation (No. 141003). S. Lee is supported in part by NRF Grant 2012008373 (Republic of Korea). The second named author would like to thank J. Bourgain for a discussion about the restriction estimate and his encouragement.

\section{REFERENCES}

[1] B. Barcelo, On the restriction of the Fourier transform to a conical surface, Trans. Amer. Math. Soc. 292 (1985), 321-333.

[2] J. Bennett, A. Carbery and T. Tao, On the multilinear restriction and Kakeya conjectures, Acta Math. 196 (2006), 261-302.

[3] J. Bourgain, Besicovitch type maximal operators and applications to Fourier analysis, Geom. Funct. Anal. 1 (1991), 147-187.

[4] J. Bourgain and L. Guth, Bounds on oscillatory integral operators based on multilinear estimates, Geom. Funct. Anal. 21 (2011), 1239-1295.

[5] Y. Cho and S. Lee, Strichartz estimates in spherical coordinates, Indiana Univ. Math. J. 62 (2013), 991-1020.

[6] J. Jiang, C. Wang and X. Yu, Generalized and weighted Strichartz estimates, Communications on Pure and Applied Analysis 11 (2012), 1723-1752.

[7] C. Fefferman, Inequalities for strongly singular convolution operators, Acta Math. 124 (1970), 9-36.

[8] J. Garcia-Cuerva and J.L. Rubio de Francia, Weighted norm inequality and related topics, North-Holland, 1985.

[9] Z. Guo, S. Lee, K. Nakanishi and C. Wang, Generalized Strichartz Estimates and Scattering for 3D Zakharov System, Comm. Math. Phys. 331 (2014), 239-259.

[10] L. Guth, A restriction estimate using polynomial partitioning, arXiv:1407.1916.

[11] L. Hörmander, The analysis of linear partial differential operators I: Distribution Theory and Fourier Analysis, 2nd ed., Springer-Verlag, 1990.

[12] M. Keel and T. Tao, Endpoint Strichartz estimates, Amer. J. Math. 120 (1998), 955-980. 
[13] S. Lee, Bilinear restriction estimates for surfaces with curvatures of different signs, Trans. Amer. Math. Soc. 358 (2006), 3511-3533.

[14] S. Lee and A. Vargas, Restriction estimates for some surfaces with vanishing curvatures, J. Funct. Anal. 258 (2010), 2884-2909.

[15] S. Lee, K. Rogers and A. Seeger, On space-time estimates for the Schrödinger operator, J. Math. Pures Appl. (9) 99 (2013), 62-85.

[16] S. Roudenko, Matrix-weighted Besov Spaces, Trans. Amer. Math. Soc 355 (2002), 273-314.

[17] E. M. Stein, Oscillatory integrals in Fourier analysis, Beijing Lectures in Harmonic Analysis, Annals of Math. Study 112, 307-355, Princeton Univ. Press (1986).

[18] — Harmonic analysis: real-variable methods, orthogonality, and oscillatory integrals, Princeton University Press, 1993.

[19] J. Sterbenz, Angular regularity and Strichartz estimates for the wave equation, with an appendix by Igor Rodnianski, Int. Math. Res. Not. 2005, no.4, 187-231.

[20] M. E. Taylor, Partial differential equations 1: Basic theory, 2nd ed., Springer, 2011.

[21] T. Tao, A Sharp bilinear restriction estimate for paraboloids, Geom. Funct. Anal. 13 (2003), $1359-1384$.

[22] T. Tao and A. Vargas, A bilinear approach to cone multipliers I. Restriction estimates, Geom. Funct. Anal. 10 (2000), 185-215.

[23] T. Tao, A. Vargas and L. Vega, A bilinear approach to the restriction and Kakeya conjecture, J. Amer. Math. Soc. 11 (1998), 967-1000.

[24] P. Tomas, A restriction theorem for the Fourier transform, Bull. Amer. Math. Soc. 81 (1975), $477-478$

[25] H. Triebel, Theory of Function Spaces, Birkhäuser, 1983.

[26] A. Vargas, Restriction theorems for a surface with negative curvature, Math. Z. 249 (2005), $97-111$.

[27] T. Wolff, An improved bound for Kakeya type maximal functions, Rev. Mat. Iberoamericana 11 (1995), 651-674.

[28] _ A sharp cone restriction estimate, Annals of Math. 153 (2001), 661-698.

[29] A. Zygmund, On Fourier coefficients and transforms of functions of two variables, Studia Math. 50 (1974), 189-201.

Yonggeun Cho, Department of Mathematics, and Institute of Pure and Applied Mathematics, Chonbuk National University, Jeonju 561-756, Republic of Korea

E-mail address: changocho@jbnu.ac.kr

Zihua Guo, School of Mathematical Sciences, Peking University, Beijing 100871, China

E-mail address: zihuaguo@math.pku.edu.cn

Sanghyuk lee, Department of Mathematical Sciences, Seoul National UniverSity, Seoul 151-747, Republic of Korea

E-mail address: shklee@snu.ac.kr 